\title{
ON SOME TOPOLOGICAL PROPERTIES IN GRADUAL NORMED SPACES *
}

\author{
Mina Ettefagh, Farnaz Y. Azari and Sina Etemad
}

\begin{abstract}
(C) by University of Niš, Serbia | Creative Commons Licence: CC BY-NC-ND Abstract. In this paper, we have investigated some topological properties of sets in a given gradual normed space. We have stated gradual Hausdorff property and then, we have studied the relationship between gradual closed sets and gradual compact sets. Also, we have given a result about having the closure point for an infinite set in a gradual normed space. In the end, we have provided some illustrative examples.
\end{abstract}

Keywords: gradual normed space; Hausdorff property; gradual numbers.

\section{Introduction}

In 1965, Zadeh first introduced a new class of sets named fuzzy sets to quantify some linguistic terms and stated these terms mathematically [12]. Indeed, fuzzy sets are generalization of classical sets and also, under certain conditions, we consider a fuzzy subset as a fuzzy number. But, when we study this notion in fuzzy metric spaces, the term fuzzy number is used instead of fuzzy intervals.

From this point of view, fuzzy numbers are generalization of intervals, not numbers. On the other hand, some algebraic properties of numbers not hold for fuzzy numbers. These problems have been implied to avoid confusion between the researchers.

In this way, in 2006, Fortin, Dubois and Fargier introduced gradual numbers as elements of fuzzy intervals [5]. In this new structure, gradual numbers are considered as an unique generalization of real numbers which are equipped with all algebraic properties of classical real numbers [5]. Since then, gradual numbers have been applied as a strong tool for computations and optimization problems.

In [7], Kasperski et al. investigated gradual numbers and applied this notion to solving combinatorial optimization problems. Some years later, Fortin et al. [6] suggested some methods for evaluating the optimality by using gradual numbers.

Received May 19, 2018; accepted November 24, 2019

2010 Mathematics Subject Classification. Primary 03E72; Secondary 28E10 
For more details about other applications of gradual numbers, see ([1], [2], [8], [11], [13], [14]).

Recently, Sadeqi and Azari [9] have studied some properties of gradual numbers and introduced gradual normed linear space. In [4], Ettefagh et al. investigated some properties of sequences in gradual normed spaces.

Motivated by above works, in this paper, we have investigated some topological properties of sets in a given gradual normed space. We have stated gradual Hausdorff property and then, we have studied the relationship between gradual closed sets and gradual compact sets. Also, we have given a result about having the closure point for an infinite set in a gradual normed space. Finally, in the last section, we have presented some illustrative examples.

\section{Preliminaries}

In this section, we recall some basic definitions and theorems on the gradual numbers and gradual normed space. For more details, see ([3], [5], [9]).

Definition 2.1. ([5]) A gradual real number $\tilde{r}$ is defined by an assignment function $A_{\tilde{r}}$ from $(0,1]$ to the set of real numbers $\mathbb{R}$. The set of all gradual real numbers is denoted by $G(\mathbb{R})$. We say that a gradual real number $\tilde{r}$ is non-negative if for each $\alpha \in(0,1], A_{\tilde{r}}(\alpha) \geq 0$. The set of all non-negative gradual real numbers is denoted by $G^{*}(\mathbb{R})$.

The gradual operations on the elements of $G(\mathbb{R})$ can be defined as follows.

Definition 2.2. ([5]) Assume that $*$ is any operation in real numbers and $\tilde{r}_{1}$ and $\tilde{r}_{2}$ are two arbitrary gradual numbers with assignment functions $A_{\tilde{r}_{1}}$ and $A_{\tilde{r}_{2}}$, respectively. Then $\tilde{r}_{1} * \tilde{r}_{2}$ is the gradual number with an assignment function $A_{\tilde{r}_{1} * \tilde{r}_{2}}$ given by

$$
A_{\tilde{r}_{1} * \tilde{r}_{2}}(\alpha)=A_{\tilde{r}_{1}}(\alpha) * A_{\tilde{r}_{2}}(\alpha), \quad(\alpha \in(0,1]) .
$$

Then, the gradual addition $\tilde{r}_{1}+\tilde{r}_{2}$ and the gradual scalar multiplication $c \tilde{r}(c \in \mathbb{R})$ are defined by

$$
A_{\tilde{r}_{1}+\tilde{r}_{2}}(\alpha)=A_{\tilde{r}_{1}}(\alpha)+A_{\tilde{r}_{2}}(\alpha), \quad A_{c \tilde{r}}(\alpha)=c A_{\tilde{r}}(\alpha),
$$

for each $\alpha \in(0,1]$.

For each real number $t \in \mathbb{R}$, the constant gradual number $\tilde{t}$ is defined by $A_{\tilde{t}}(\alpha)=t$ for each $\alpha \in(0,1]$. In particular, $\tilde{0}$ and $\tilde{1}$ are constant gradual numbers defined by $A_{\tilde{0}}(\alpha)=0$ and $A_{\tilde{1}}(\alpha)=1$, respectively. It can be easily proved that $G(\mathbb{R})$ with the gradual addition and gradual scalar multiplication is a real linear space [5].

Definition 2.3. ([7]) Let $\tilde{r}, \tilde{s} \in G(\mathbb{R})$. The partial order relation $\leq$ in $G(\mathbb{R})$ is defined by $\tilde{r} \leq \tilde{s}$ if and only if $A_{\tilde{r}}(\alpha) \leq A_{\tilde{s}}(\alpha)$ for all $\alpha \in(0,1]$. 
Theorem 2.1. ([r]) Let $\tilde{r}, \tilde{s}, \tilde{t} \in G(\mathbb{R})$. We have

(i) if $\tilde{r} \leq \tilde{s}$, then $\tilde{r}-\tilde{t} \leq \tilde{s}-\tilde{t}$;

(ii) if $\tilde{r} \leq \tilde{s}$ and $\tilde{0} \leq \tilde{t}$, then $\tilde{r} \cdot \tilde{t} \leq \tilde{s} \cdot \tilde{t}$ and $\frac{\tilde{r}}{\tilde{t}} \leq \frac{\tilde{s}}{\tilde{t}}, \tilde{t} \neq \tilde{0}$;

(iii) $\frac{(\tilde{r} \cdot \tilde{s})}{\tilde{t}}=\tilde{r} \cdot\left(\frac{\tilde{s}}{\tilde{t}}\right), \tilde{t} \neq \tilde{0}$.

Definition 2.4. ([9]) Let $X$ be a real vector space and $x, y \in X$. The mapping $\|\cdot\|_{G}$ from $X$ to $G^{*}(\mathbb{R})$ is called a gradual norm on $X$ if for each $\alpha \in(0,1]$, we have

(G1) $A_{\|x\|_{G}}(\alpha)=A_{\tilde{0}}(\alpha)$ iff $x=0$;

(G2) $A_{\|k x\|_{G}}(\alpha)=|k| A_{\|x\|_{G}}(\alpha) ; \quad(k \in \mathbb{R})$

(G3) $A_{\|x+y\|_{G}}(\alpha) \leq A_{\|x\|_{G}}(\alpha)+A_{\|y\|_{G}}(\alpha)$.

Then the pair $\left(X,\|\cdot\|_{G}\right)$ is called a gradual normed space (GNS).

Example 2.1. ([9]) (i) Let $X=\mathbb{R}^{n}$. For each $\alpha \in(0,1]$ and $x=\left(x_{1}, \ldots, x_{n}\right) \in \mathbb{R}^{n}$, consider the function $\|\cdot\|_{G}: \mathbb{R}^{n} \rightarrow G^{*}(\mathbb{R})$ by

$$
A_{\|x\|_{G}}(\alpha)=e^{\alpha} \sum_{i=1}^{n}\left|x_{i}\right|
$$

Then $\|\cdot\|_{G}$ is a gradual norm on $\mathbb{R}^{n}$ and $\left(\mathbb{R}^{n},\|\cdot\|_{G}\right)$ is a gradual normed linear space. (ii) Let $X=C([0,1])$ be the space of all continuous real-valued functions on $[0,1]$. Consider two norms on $C([0,1])$ by $\|f\|_{0}=\left(\int_{0}^{1}|f(t)|^{2} \mathrm{~d} t\right)^{\frac{1}{2}}$ and $\|f\|_{1}=\max _{0 \leq t \leq 1}\{|f(t)|\}$. Now, the function $\|\cdot\|_{G}: C[0,1] \rightarrow G^{*}(\mathbb{R})$ defined by

$$
A_{\|f\|_{G}}(\lambda)= \begin{cases}\|f\|_{0}, & 0<\lambda \leq \frac{1}{2} \\ \|f\|_{1}, & \frac{1}{2}<\lambda \leq 1\end{cases}
$$

is a gradual norm on $X$.

Definition 2.5. ([9]) Let $X$ be a gradual normed space. A gradual neighborhood of $x_{0} \in X$ with radius of $\epsilon>0$ is defined by

$$
x_{0}+N(\alpha, \epsilon)=\left\{x: A_{\left\|x-x_{0}\right\|_{G}}(\alpha)<\epsilon\right\}, \quad \alpha \in(0,1] .
$$

In particular, if $x_{0}=0$, then $N(\alpha, \epsilon)=\left\{x: A_{\|x\|_{G}}(\alpha)<\epsilon\right\}$.

Lemma 2.1. ([9]) Let $X$ be a gradual normed space and $\alpha \in(0,1]$ and $\epsilon>0$. We have 
(N1) $N(\alpha, \epsilon)=\epsilon N(\alpha, 1)$;

(N2) if $\epsilon_{1} \leq \epsilon_{2}$, then $N\left(\alpha, \epsilon_{1}\right) \subseteq N\left(\alpha, \epsilon_{2}\right)$;

(N3) if for every $x \in X$, the assignment function $A_{\|x\|_{G}}$ be decreasing and $\alpha_{1} \leq \alpha_{2}$, then $N\left(\alpha_{1}, \epsilon\right) \subseteq N\left(\alpha_{2}, \epsilon\right)$.

Definition 2.6. ([9]) Let $X$ be a gradual normed space and $A \subseteq X$. Then

(H1) the point $x_{0} \in X$ is called a closure point of $A$ if for each $\alpha \in(0,1]$, we have

$$
\left(x_{0}+N(\alpha, \alpha)\right) \cap A \neq \varnothing .
$$

The set of all closure points of $A$ is denoted by $\bar{A}$.

(H2) The point $x_{0} \in X$ is called a limit point of $A$ if for each $\alpha \in(0,1]$, $\left(x_{0}+N(\alpha, \alpha)\right) \cap A$ contains at least one point of $A$ different from $x_{0}$ itself, or

$$
\left(x_{0}+N(\alpha, \alpha)\right)^{*} \cap A \neq \varnothing,
$$

where $\left(x_{0}+N(\alpha, \alpha)\right)^{*}=\left(x_{0}+N(\alpha, \alpha)\right) \backslash\left\{x_{0}\right\}$.

(H3) the point $x_{0} \in A$ is called an interior point of $A$ if there exists $N\left(\alpha_{0}, \epsilon_{0}\right)$ such that $x_{0}+N\left(\alpha_{0}, \epsilon_{0}\right) \subseteq A$. The set of all interior points of $A$ is denoted by Int $A$.

(H4) the set $A$ is said to be gradual closed set iff $\bar{A}=A$.

(H5) the set $A$ is said to be gradual open set iff $\operatorname{Int} A=A$.

The following theorems state an effective relationship between gradual open sets and gradual closed sets.

Theorem 2.2. Let $\left(X,\|\cdot\|_{G}\right)$ be a gradual normed space and for every $x \in X$, $A_{\|x\|_{G}}$ be a decreasing function. If $B$ is the gradual open subset of $X$, then $X \backslash B$ is gradual closed.

Proof. Let $B$ be a gradual open set and $x_{0}$ be a closure point of $X \backslash B$. Then for each $\alpha \in(0,1]$, we have $\left(x_{0}+N(\alpha, \alpha)\right) \cap(X \backslash B) \neq \varnothing$ and so $\left(x_{0}+N(\alpha, \alpha)\right) \nsubseteq B$. Now, for each $\epsilon>0$ and $\alpha \in(0,1]$, let $\alpha_{0}=\min \{\alpha, \epsilon\}$. Thus we have

$$
\left(x_{0}+N\left(\alpha_{0}, \alpha_{0}\right)\right) \subseteq\left(x_{0}+N(\alpha, \epsilon)\right)
$$

and then $\left(x_{0}+N(\alpha, \epsilon)\right) \nsubseteq B$. Hence $x_{0} \notin \operatorname{Int} B=B$ or $x_{0} \in X \backslash B$ and we conclude that $X \backslash B$ is gradual closed.

Theorem 2.3. Let $\left(X,\|\cdot\|_{G}\right)$ be a gradual normed space. For every subset $B$ of $X$, if $X \backslash B$ is the gradual closed set, then $B$ is gradual open set.

Proof. Suppose that $X \backslash B$ is a gradual closed set. Then $\overline{X \backslash B}=X \backslash B$. Let $x_{0} \in B$, thus $x_{0} \notin X \backslash B=\overline{X \backslash B}$. Hence for some $\alpha \in(0,1]$,

$$
\left(x_{0}+N(\alpha, \alpha)\right) \cap(X \backslash B)=\varnothing,
$$

or $\left(x_{0}+N(\alpha, \alpha)\right) \subseteq B$. We conclude that $x_{0}$ is an interior point for $B$ and $B$ is a gradual open set. 


\section{Main Results}

Now, in this section, we are ready to state main results.

Theorem 3.1. Let $\left(X,\|\cdot\|_{G}\right)$ be a gradual normed space and $A$ and $B$ be subsets of $X$. Then

(i) $(\overline{A \cap B}) \subseteq \bar{A} \cap \bar{B}$ and $(\overline{A \cup B})=\bar{A} \cup \bar{B}$.

(ii) if every assignment function is decreasing, we have

$$
\operatorname{Int}(A) \cup \operatorname{Int}(B) \subseteq \operatorname{Int}(A \cup B) \text { and } \operatorname{Int}(A \cap B)=\operatorname{Int}(A) \cap \operatorname{Int}(B) .
$$

Proof. (i) Let $x \in(\overline{A \cap B})$. Then for every $\alpha \in(0,1]$, we have

$$
(x+N(\alpha, \alpha)) \cap(A \cap B) \neq \varnothing .
$$

So for every $\alpha \in(0,1]$, we have $(x+N(\alpha, \alpha)) \cap A \neq \varnothing$ and $(x+N(\alpha, \alpha)) \cap B \neq \varnothing$. This shows that $x \in \bar{A} \cap \bar{B}$.

Also, $x \in(\overline{A \cup B})$ if and only if for every $\alpha \in(0,1]$, we have

$$
(x+N(\alpha, \alpha)) \cap(A \cup B) \neq \varnothing .
$$

Thus, one can write $(x+N(\alpha, \alpha)) \cap A \neq \varnothing$ or $(x+N(\alpha, \alpha)) \cap B \neq \varnothing$. This means that $x \in \bar{A} \cup \bar{B}$.

(ii) Let $x \in \operatorname{Int}(A) \cup \operatorname{Int}(B)$. Then $x \in \operatorname{Int}(A)$ or $x \in \operatorname{Int}(B)$. So for some $\alpha_{1}, \alpha_{2} \in(0,1]$ and $\epsilon_{1}, \epsilon_{2}>0$, we have

$$
\left(x+N\left(\alpha_{1}, \epsilon_{1}\right)\right) \subset A \text { or }\left(x+N\left(\alpha_{2}, \epsilon_{2}\right)\right) \subset B .
$$

Now, let $\alpha<\min \left\{\alpha_{1}, \alpha_{2}\right\}$ and $\epsilon<\min \left\{\epsilon_{1}, \epsilon_{2}\right\}$. Since every assignment function is decreasing, thus $(x+N(\alpha, \epsilon)) \subset(A \cup B)$ and we get $x \in \operatorname{Int}(A \cup B)$.

One can similarly prove the equality $\operatorname{Int}(A \cap B)=\operatorname{Int}(A) \cap \operatorname{Int}(B)$ and we omit this part of the proof.

Theorem 3.2. Let $\left(X,\|\cdot\|_{G}\right)$ be a gradual normed space. Then

(i) for any collection $\left\{G_{\gamma}\right\}_{\gamma}$ of gradual open sets, $\bigcup_{\gamma} G_{\gamma}$ is a gradual open set.

(ii) for any collection $\left\{F_{\gamma}\right\}_{\gamma}$ of gradual closed sets, $\bigcap_{\gamma} F_{\gamma}$ is a gradual closed set.

Proof. (i) Let $\left\{G_{\gamma}\right\}_{\gamma}$ be an arbitrary collection of gradual open sets and let $x \in \bigcup_{\gamma} G_{\gamma}$. Then, there is some $\gamma_{0}$ such that $x \in G_{\gamma_{0}}$. Since $G_{\gamma_{0}}$ is a gradual open set, thus there exists $\alpha \in(0,1]$ and $\epsilon>0$ such that

$$
(x+N(\alpha, \epsilon)) \subseteq G_{\gamma_{0}} \subseteq \bigcup_{\gamma} G_{\gamma} .
$$


Consequently, $\bigcup_{\gamma} G_{\gamma}$ is a gradual open set.

(ii) Let $\left\{F_{\gamma}\right\}_{\gamma}$ be an arbitrary collection of gradual closed sets and put $F=\bigcap_{\gamma} F_{\gamma}$. If $x \in \bar{F}$, then for each $\alpha \in(0,1]$, we have $(x+N(\alpha, \alpha)) \cap F \neq \varnothing$. Hence for every $\gamma$, we have $(x+N(\alpha, \alpha)) \cap F_{\gamma} \neq \varnothing$ and so $x \in \bar{F}_{\gamma}$. On the other hand, since for each $\gamma, F_{\gamma}$ is a gradual closed set, so $x \in \bar{F}_{\gamma}$ implies that $x \in F_{\gamma}$. Therefore $x \in \bigcap_{\gamma} F_{\gamma}=F$. This shows that $\bar{F} \subseteq F$ and so $F$ is a gradual closed set.

Now, for the finite number of gradual open (closed) sets, we have the following theorem.

Theorem 3.3. Let $\left(X,\|\cdot\|_{G}\right)$ be a gradual normed space. Hence

(i) if every assignment function is decreasing, then for any finite collection $G_{1}, G_{2}, \cdots, G_{n}$ of gradual open sets, $\bigcap_{i=1}^{n} G_{i}$ is a gradual open set.

(ii) for any finite collection $F_{1}, F_{2}, \cdots, F_{n}$ of gradual closed sets, $\bigcup_{i=1}^{n} F_{i}$ is a gradual closed set.

Proof. (i) Let $G_{1}, G_{2}, \cdots, G_{n}$ be a finite collection of gradual open sets and let $x \in \bigcap_{i=1}^{n} G_{i}$ be an arbitrary element. Then for any $1 \leq i \leq n, x \in G_{i}$. But every $G_{i}$ is a gradual open set, thus for each $1 \leq i \leq n$, there are $\alpha_{i} \in(0,1]$ and $\epsilon_{i}>0$ such that $\left(x+N\left(\alpha_{i}, \epsilon_{i}\right)\right) \subseteq G_{i}$. Put $\alpha<\min \left\{\alpha_{1}, \alpha_{2}, \cdots, \alpha_{n}\right\}$ and $\epsilon<\min \left\{\epsilon_{1}, \epsilon_{2}, \cdots, \epsilon_{n}\right\}$. Since for each $x \in X$, the assignment function $A_{\|x\|_{G}}$ is decreasing, so we have $N(\alpha, \epsilon) \subseteq N\left(\alpha_{i}, \epsilon_{i}\right)$, for all $1 \leq i \leq n$. Thus

$$
(x+N(\alpha, \epsilon)) \subseteq\left(x+N\left(\alpha_{i}, \epsilon_{i}\right)\right) \subseteq G_{i} .
$$

Therefore, $(x+N(\alpha, \epsilon)) \subseteq \bigcap_{i=1}^{n} G_{i}$. Hence $\bigcap_{i=1}^{n} G_{i}$ is a gradual open set.

(ii) Since each $F_{i}$ is gradual closed, thus $\bar{F}_{i}=F_{i}$. Now, suppose that $x_{0} \in \overline{\bigcup_{i=1}^{n} F_{i}}$, so for each $\alpha \in(0,1]$, we have

$$
\left(x_{0}+N(\alpha, \alpha)\right) \cap\left(\bigcup_{i=1}^{n} F_{i}\right) \neq \varnothing .
$$

This means that for each $\alpha \in(0,1]$, there exists $1 \leq i \leq n$ with

$$
\left(x_{0}+N(\alpha, \alpha)\right) \cap F_{i} \neq \varnothing,
$$

and so $x_{0} \in \bar{F}_{i}=F_{i}$. Therefore, $x_{0} \in \bigcup_{i=1}^{n} F_{i}$. Hence, we conclude that $\overline{\bigcup_{i=1}^{n} F_{i}} \subseteq \bigcup_{i=1}^{n} F_{i}$ and the proof is completed.

Theorem 3.4. (Gradual Hausdorff property) Let $\left(X,\|\cdot\|_{G}\right)$ be a gradual normed space and $x, y \in X$ with $x \neq y$. Then there exists two disjoint neighborhoods of $x$ and $y$. 
Proof. Since $x \neq y$, so for each $\alpha \in(0,1]$, we have $A_{\|x-y\|_{G}}(\alpha) \neq A_{\tilde{0}}(\alpha)$. Thus, there is $\alpha_{0} \in(0,1]$ such that $A_{\|x-y\|_{G}}\left(\alpha_{0}\right)>0$. Put $\epsilon_{0}<\frac{1}{2} A_{\|x-y\|_{G}}\left(\alpha_{0}\right)$ and we claim that $\left(x+N\left(\alpha_{0}, \epsilon_{0}\right)\right) \cap\left(y+N\left(\alpha_{0}, \epsilon_{0}\right)\right)=\varnothing$. To prove this claim, let $z \in\left(x+N\left(\alpha_{0}, \epsilon_{0}\right)\right) \cap\left(y+N\left(\alpha_{0}, \epsilon_{0}\right)\right)$. Then, we have $A_{\|x-z\|_{G}}\left(\alpha_{0}\right)<\epsilon_{0}$ and $A_{\|y-z\|_{G}}\left(\alpha_{0}\right)<\epsilon_{0}$. Now, one can write

$$
\begin{aligned}
A_{\|x-y\|_{G}}\left(\alpha_{0}\right) & \leq A_{\|x-z\|_{G}}\left(\alpha_{0}\right)+A_{\|y-z\|_{G}}\left(\alpha_{0}\right) \\
& <2 \epsilon_{0}<A_{\|x-y\|_{G}}\left(\alpha_{0}\right)
\end{aligned}
$$

and this is a contradiction.

Theorem 3.5. Let $B$ be a subset of the gradual normed space $\left(X,\|\cdot\|_{G}\right)$ and for each $x \in X$, the assignment function $A_{\|x\|_{G}}$ be decreasing. If $x_{0}$ is a limit point of $B$, then every neighborhood of $x_{0}$ contains infinitely many points of $B$.

Proof. Suppose that there exists $\alpha \in(o, 1]$ and $\epsilon>0$ such that the neighborhood $\left(x_{0}+N(\alpha, \epsilon)\right)$ of $x_{0}$ contains only finite number of elements of $B$, i.e. $\left(x_{0}+N(\alpha, \epsilon)\right) \cap B=\left\{y_{1}, y_{2}, \ldots, y_{n}\right\}$. Put

$$
\epsilon_{0}<\min \left\{A_{\left\|x_{0}-y_{1}\right\|_{G}}(\alpha), A_{\left\|x_{0}-y_{2}\right\|_{G}}(\alpha), \ldots, A_{\left\|x_{0}-y_{n}\right\|_{G}}(\alpha)\right\} .
$$

Since $\epsilon_{0}<\epsilon$, hence $\left(x_{0}+N\left(\alpha, \epsilon_{0}\right)\right) \subset\left(x_{0}+N(\alpha, \epsilon)\right)$. Now, we claim that the neighborhood $\left(x_{0}+N\left(\alpha, \epsilon_{0}\right)\right)$ contains no point of $B$. Indeed, if for some $i(i=1,2, \ldots, n), y_{i} \in\left(x_{0}+N\left(\alpha, \epsilon_{0}\right)\right)$, then we have

$$
A_{\left\|x_{0}-y_{i}\right\|_{G}}(\alpha)<\epsilon_{0}<A_{\left\|x_{0}-y_{i}\right\|_{G}}(\alpha)
$$

and this is a contradiction. Therefore $\left(x_{0}+N\left(\alpha, \epsilon_{0}\right)\right)^{*} \cap B=\varnothing$. Now, put $\alpha_{0}<\min \left\{\epsilon_{0}, \alpha\right\}$. So, we have $\alpha_{0}<\epsilon_{0}$ and $\alpha_{0}<\alpha$. Since every assignment function is decreasing, thus

$$
\left(x_{0}+N\left(\alpha_{0}, \alpha_{0}\right)\right) \subset\left(x_{0}+N\left(\alpha, \epsilon_{0}\right)\right) .
$$

Hence, it is followed that $\left(x_{0}+N\left(\alpha_{0}, \alpha_{0}\right)\right)^{*} \cap B=\varnothing$, which contradicts to the being limit point $x_{0}$ for $B$ and the proof is completed.

Now, we define the new concept "gradual compact set" in a gradual normed space.

Definition 3.1. Let $\left(X,\|\cdot\|_{G}\right)$ be a gradual normed space and $K$ be an arbitrary nonempty subset of $X$. We say that $K$ is a gradual compact set if for each cover $\left\{V_{i}\right\}_{i \in I}$ of gradual open sets for $K$, there exists finite number $V_{i}(i=1, \cdots, n)$ such that $K \subseteq \bigcup_{i=1}^{n} V_{i}$.

Theorem 3.6. Let $\left(X,\|\cdot\|_{G}\right)$ be a gradual normed space and for each $x \in X$, the assignment function $A_{\|x\|_{G}}$ be decreasing. Then every gradual compact set is gradual closed. 
Proof. Let $K$ be a gradual compact subset of $X$. We will show that $X \backslash K$ is a gradual open set. For this, assume that $p \in(X \backslash K)$ and for each $q \in K$, consider neighborhoods $V_{q}=\left(p+N\left(\alpha_{0}, \epsilon\right)\right)$ and $W_{q}=\left(q+N\left(\alpha_{0}, \epsilon\right)\right)$ for $p$ and $q$, respectively, where $\alpha_{0} \in(0,1]$ is a fixed number and

$$
\epsilon<\frac{1}{2} A_{\|p-q\|_{G}}\left(\alpha_{0}\right) .
$$

For each $q \in K$, we have $V_{q} \cap W_{q}=\varnothing$; because if $z \in V_{q} \cap W_{q}$, then we get $A_{\|z-p\|_{G}}\left(\alpha_{0}\right)<\epsilon$ and $A_{\|z-q\|_{G}}\left(\alpha_{0}\right)<\epsilon$. So

$$
A_{\|p-q\|_{G}}\left(\alpha_{0}\right)<2 \epsilon<A_{\|p-q\|_{G}}\left(\alpha_{0}\right)
$$

which is a contradiction.

Now, the cover $\bigcup_{q \in K} W_{q}$ of gradual open sets for $K$ has finite subcover as follows:

$$
\exists q_{1}, q_{2}, \cdots, q_{n} \in K \quad \text { s.t. } K \subseteq \bigcup_{i=1}^{n} W_{q_{i}} .
$$

Let $V=\bigcap_{i=1}^{n} V_{q_{i}}$. Since $V$ is a finite intersection of gradual open sets containing $p$ and each assignment function is decreasing, so by Theorem 3.3 (i), $V$ is a gradual open set containing $p$. Therefore $V \cap K=\varnothing$ and so $V \subseteq(X \backslash K)$. This proves that $X \backslash K$ is a gradual open set and by Theorem $2.2, K$ is a gradual closed set.

Theorem 3.7. Let $\left(X,\|\cdot\|_{G}\right)$ be a gradual normed space and $K$ be a gradual compact subset of $X$. If $F$ is a gradual closed subset of $K$, then $F$ is gradual compact.

Proof. Suppose that $\left\{V_{i}\right\}_{i \in I}$ is a cover of gradual open subsets for $F$. By Theorem 2.3, $X \backslash F$ is a gradual open subset and so $\left\{V_{i}\right\}_{i \in I} \cup(X \backslash F)$ is a cover for $K$. Since $K$ is gradual compact, there exists a finite cover $\left\{V_{i}\right\}_{i=1}^{n} \cup(X \backslash F)$ for $K$. This implies that $\left\{V_{i}\right\}_{i=1}^{n}$ is a finite cover for $F$. Hence $F$ will be a gradual compact set.

Corollary 3.1. Let $\left(X,\|\cdot\|_{G}\right)$ be a gradual normed space such that every assignment function is decreasing. Suppose that $F$ and $K$ are gradual closed and gradual compact subsets of $X$, respectively. Then $F \cap K$ is a gradual compact set.

Proof. This is a consequence of Theorems 3.6, 3.7 and 3.2(ii).

Finally, we give the last result about having closure point for an infinite set in a gradual normed space. This property is like to Bolzano-Weierstrass property in metric spaces [10].

Theorem 3.8. Let $\left(X,\|\cdot\|_{G}\right)$ be a gradual normed space and $E$ be an infinite subset of the gradual compact set $K$. Then $E$ has the limit point in $K$. 
Proof. Suppose that every $x \in K$ is not a limit point of $E$. Then there exists $\alpha_{x} \in(0,1]$ such that $\left(x+N\left(\alpha_{x}, \alpha_{x}\right)\right)^{*} \cap E=\varnothing$. Hence, if $x \in E$ is arbitrary, then $\left(x+N\left(\alpha_{x}, \alpha_{x}\right)\right) \cap E=\{x\}$. This shows that there is no finite collection of infinite cover $\left\{x+N\left(\alpha_{x}, \alpha_{x}\right)\right\}_{x \in E}$ of gradual open sets for $E$ which covers the set $E$. Now, let $\left\{G_{i}\right\}_{i \in I}$ be an arbitrary cover of gradual open sets for $K \backslash E$. Then

$$
\left\{x+N\left(\alpha_{x}, \alpha_{x}\right)\right\}_{x \in E} \cup\left\{G_{i}\right\}_{i \in I}
$$

is a cover of gradual open sets for $K$ which contains no finite subcover and this contradicts to the gradual compactness of $K$.

\section{Examples}

The following examples are generalizations of the Example 2.1. Also we have some arguments about gradual interior and gradual closure points in each example.

Example 4.1. Let $(X,\|\cdot\|)$ be a real normed space. We define the function $\|\cdot\|_{G}: X \rightarrow G^{*}(\mathbb{R})$ by

$$
A_{\|x\|_{G}}(\alpha)=f(\alpha)\|x\| ; \quad(\alpha \in(0,1], x \in X)
$$

where $f:(0,1] \rightarrow \mathbb{R}^{+}$is a nonzero function. One can easily verify that $\|\cdot\|_{G}$ is a gradual norm on $X$. Also, if we denote the neighborhoods in $(X,\|\cdot\|)$ by

$$
N_{\epsilon}(x)=\{a \in X:\|x-a\|<\epsilon\}, \quad(\epsilon>0),
$$

then in gradual normed space $\left(X,\|\cdot\|_{G}\right)$, for $\epsilon>0$ and $\alpha \in(0,1]$ we have

$$
N(\alpha, \epsilon)=N_{\frac{\epsilon}{f(\alpha)}}(0)
$$

and for $x \in X,(x+N(\alpha, \epsilon))=N_{\frac{\epsilon}{f(\alpha)}}(x)$.

Hence we conclude that $\operatorname{Int}(A)=\operatorname{Int}_{G}(A)$, where $\operatorname{Int}(A)$ and $\operatorname{Int}_{G}(A)$ denote the set of all interior points of $A$ in $(X,\|\cdot\|)$ and $\left(X,\|\cdot\|_{G}\right)$, respectively.

Now, suppose that $A \subset X$ and $x \in X$ is a closure point of $A$ in $(X,\|\cdot\|)$. Then for every $\epsilon>0$, we have $N_{\epsilon}(x) \cap A \neq \varnothing$. Thus for every $\alpha \in(0,1]$ and $\epsilon=\frac{\alpha}{f(\alpha)}$, we can write

$$
(x+N(\alpha, \alpha)) \cap A=N_{\frac{\alpha}{f(\alpha)}}(x) \cap A \neq \varnothing,
$$

and we conclude that $x$ is a closure point of $A$ in $\left(X,\|\cdot\|_{G}\right)$ or $\bar{A} \subset \bar{A}^{G}$, in which $\bar{A}$ and $\bar{A}^{G}$ denote the closure of $A$ in $(X,\|\cdot\|)$ and $\left(X,\|\cdot\|_{G}\right)$, respectively.

Example 4.2. Let $\|\cdot\|_{1}$ and $\|\cdot\|_{2}$ be two norms on real vector space $X$ such that they are not equivalent norms. For $x \in X$ and $\alpha \in(0,1]$, we define the function $\|\cdot\|_{G}: X \rightarrow G^{*}(\mathbb{R})$ by

$$
A_{\|x\|_{G}}(\alpha)= \begin{cases}\|x\|_{1}, & 0<\alpha \leq \frac{1}{2} \\ \|x\|_{2}, & \frac{1}{2}<\alpha \leq 1\end{cases}
$$


It is easy to check that $\left(X,\|\cdot\|_{G}\right)$ will be a gradual normed space. This example can be extended to a finite number of non-equivalent norms.

Now, for $x \in X$ and $\epsilon>0$, let

$$
\begin{aligned}
& { }_{1} N_{\epsilon}(x)=\left\{a \in X:\|x-a\|_{1}<\epsilon\right\}, \\
& { }_{2} N_{\epsilon}(x)=\left\{a \in X:\|x-a\|_{2}<\epsilon\right\} .
\end{aligned}
$$

Therefore in $\left(X,\|\cdot\|_{G}\right)$, we can write for $\epsilon>0$ and $\alpha \in(0,1]$,

$$
N(\alpha, \epsilon)=\left\{x \in X: A_{\|x\|_{G}}(\alpha)<\epsilon\right\}= \begin{cases}{ }_{1} N_{\epsilon}(0), & 0<\alpha \leq \frac{1}{2} \\ { }_{2} N_{\epsilon}(0), & \frac{1}{2}<\alpha \leq 1\end{cases}
$$

and also for $x \in X$,

$$
(x+N(\alpha, \epsilon))=\left\{\begin{array}{cc}
{ }_{1} N_{\epsilon}(x), & 0<\alpha \leq \frac{1}{2} \\
{ }_{2} N_{\epsilon}(x), & \frac{1}{2}<\alpha \leq 1 .
\end{array}\right.
$$

Then we can conclude that

$$
\operatorname{Int}_{G}(A)=\operatorname{Int}_{1}(A) \cup \operatorname{Int}_{2}(A),
$$

where $\operatorname{Int}_{G}(A), \operatorname{Int}_{1}(A)$ and $\operatorname{Int}_{2}(A)$ denote the set of all interior points of $A$ in $\left(X,\|\cdot\|_{G}\right)$, $\left(X,\|\cdot\|_{1}\right)$ and $\left(X,\|\cdot\|_{2}\right)$, respectively.

Now, suppose that $x \in \bar{A}^{G}$. So for every $\alpha \in(0,1],(x+N(\alpha, \alpha)) \cap A \neq \varnothing$; In particular, it will be true for each $\alpha \in\left(0, \frac{1}{2}\right]$. This shows that $\bar{A}^{G} \subset \bar{A}^{1}$, where $\bar{A}^{1}$ denote the closure of $A$ in the space $\left(X,\|\cdot\|_{1}\right)$. Finally, suppose that $x \in \bar{A}^{1} \cap \bar{A}^{2}$. Then for each $\alpha>0$, we have ${ }_{1} N_{\alpha}(x) \cap A \neq \varnothing$ and ${ }_{2} N_{\alpha}(x) \cap A \neq \varnothing$. Consequently, for each $\alpha \in\left(0, \frac{1}{2}\right]$, we have

$$
(x+N(\alpha, \alpha)) \cap A={ }_{1} N_{\alpha}(x) \cap A \neq \varnothing,
$$

and for each $\alpha \in\left(\frac{1}{2}, 1\right]$, we have

$$
(x+N(\alpha, \alpha)) \cap A={ }_{2} N_{\alpha}(x) \cap A \neq \varnothing .
$$

This shows that $x \in \bar{A}^{G}$ and we conclude that $\left(\bar{A}^{1} \cap \bar{A}^{2}\right) \subset \bar{A}^{G}$.

\section{R E F E R E N C E S}

1. F. Aiche and D. Dubois: Possibility and gradual number approaches to ranking methods for random fuzzy intervals. Communications in Computer and Information Sciences, 299 (2012) 9-18.

2. P. Brass: On the nonexistence of Hausdorff-like metrics for fuzzy sets. Pattern Recognition Letters, 23 (2002) 39-43. 
3. D. Dubois and H. Prade: Gradual elements in a fuzzy set. Soft Computing-A Fusion of Foundations, Methodologies and Applications, 12 (2007) 165-175.

4. M. Ettefagh, S. Etemad and F.Y. Azari: Some properties of sequences in gradual normed spaces. Asian-Eur. J. Math, To appear. doi:/10.1142/S1793557120500850.

5. J. Fortin, D. Dubois and H. FARGieR: Gradual numbers and their application to fuzzy interval analysis. IEEE transactions on fuzzy systems, 16 (2008) 388-402.

6. J. Fortin, A. KASPERSKi and P. ZiELinski: Some methods for evaluating the optimality of elements in matroids. Fuzzy sets and systems, 160 (2009) 1341-1354.

7. A. KASPERSKI and P. ZIELINSKI: Using gradual numbers for solving fuzzy-valued combinatorial optimization problems. Foundations of Fuzzy Logic and Soft Computing, Lecture Notes in Computer Sciences, Springer (2007) 656-665.

8. L. LIETARD and D. ROCACHER: Conditions with aggregates evaluated using gradual numbers. Control and cybernetics, 38 (2009) 395-417.

9. I. SADEQI and F. Y. AzARI: Gradual normed linear space. Iranian journal of fuzzy systems, 8 (2011) 131-139.

10. G. F. Simmons: Introduction to topology and modern analysis. Tata McGraw-Hill Education (2004) ISBN: 10:0070597847.

11. E. A. Sтоск: Gradual numbers and fuzzy optimization. PhD. Thesis, University of Colorado Denver, Denver, America (2010).

12. A. L. ZADEH: Fuzzy sets. Information \& control, 8 (1965) 338-353.

13. С. Zноч: Gradual metric spaces. Appl. Math. Sci. Vol. 9.14 (2015) 689-701.

14. C. Zhou and J. Li: New fuzzy measure based on gradual numbers, Int. J. Math. Anal. 9 (2015) 101-110.

\section{Mina Ettefagh}

Faculty of Science

Department of Mathematics

Tabriz Branch, Islamic Azad University, Tabriz, Iran

etefagh@iaut.ac.ir

Farnaz Y. Azari

Faculty of Science

Department of Mathematics

Sahand University of Technology, Tabriz, Iran

fyaqubazari@gmail.com

Sina Etemad

Department of Mathematics

Azarbaijan Shahid Madani University, Tabriz, Iran

sina.etemad@gmail.com 